\title{
Variational approach to MHD stagnation flow of nanofluid towards permeable stretching sheet
}

\author{
Muthukumaran Chandrasekar*, Malayampalayam S. Kasiviswanathan \\ Department of Mathematics, Anna University, Chennai, Tamilnadu 600025, India
}

Corresponding Author Email: mchandru@annauniv.edu

https://doi.org/10.18280/ijht.360205

Received: 7 January 2017

Accepted: 2 February 2018

\section{Keywords:}

Gyarmati's variational principle, nanofluid, stagnation flow, stretching sheet, suction / injection.

\begin{abstract}
Governing Principle of Dissipative Processes proposed by Gyarmati for non-equilibrium thermodynamics has been employed to obtain the variational solution of steady, laminar, magnetohydrodynamic stagnation flow of a nanofluid over a non-isothermal stretching sheet with Brownian motion and thermophoresis effects when the flow is controlled by suction/injection. The velocity, temperature and concentration fields inside their boundary layers are approximated by polynomial functions which are satisfied by the boundary conditions. The variational principle is formulated, and Euler-Lagrange equations of the principle are reduced to simple polynomial equations in terms of momentum, thermal and concentration boundary layer thicknesses. The temperature, concentration profiles, skin friction, heat and mass transfer effects are analyzed for various values of velocity ratio parameter $\varepsilon$, suction/injection parameter $H$, magnetic parameter $\xi$, Prandtl number $P r$, wall temperature parameter $n$, Lewis number $L e$, Brownian motion parameter $N b$ and thermophoresis parameter $N t$. The obtained results are compared with numerical solutions, and the order of accuracy is remarkable.
\end{abstract}

\section{INTRODUCTION}

In this study, the primary objective is to analyze the heat transfer enhancement in stagnation point flow towards a stretching sheet which is a combination of pioneer Hiemenz [1] and Sakiadis [2] problems.

The practical applications of this issue are in several industries where the engineering processes such as extrusion, continuous casting exist. In particular, glass blowing, drawing of metal wires, plastic films, polymer sheets and spinning of fibers, etc. In these processes, the quality of the final product solely depends on the rate of heat transfer to the stretching sheet. Hence, it is important that the analysis of heat transfer in such processes.

Crane [3] found a closed form exact solution for Sakiadis problem with the extension that of the consideration of the stretching sheet velocity is proportional to the distance from the slit.

Bhattacharya [4] has obtained variational solutions for laminar stagnation flow using Gyarmati's principle. The effect of magnetic field on stagnation point flow was investigated by Ariel [5]. The variational solution in analytical form for axisymmetric stagnation point flow using Gyarmati's principle was obtained by Antony Raj [6]. The analysis of heat transfer in stagnation flow over a stretching sheet was presented by Mahapatra and Gupta [7] and Ishak et al. [8].

In recent decades, nanofluid which is a mixture of nanosized solid particles with high thermal conductivity suspended in a conventional fluid is used to enhance the heat transfer rate in the systems involving fluid flow. Choi [9] gave the theoretical investigation about the potential benefits of nanofluids. The explanation for the abnormal convective heat transfer enhancement of nanofluid, when compared to conventional fluid was observed by Buongiorno [10].
Unsteady stagnation flow of conventional fluid past a stretching sheet was solved numerically by Sharma et al. [11] using finite difference scheme.

Bachok et al. [12] presented an analysis for laminar stagnation point flow over a stretching/shrinking sheet in a nanofluid. Ibrahim et al. [13], Ibrahim and Rizwan [14] numerically solved MHD stagnation point flow of a nanofluid past a stretching sheet with various boundary conditions. The boundary layer solution for stagnation point flow towards a stretching sheet with heat generation/ absorption and suction/injection effects was studied by Hamad and Ferdows [15]. The influence of radiation and Brownian motion, thermophoresis effects on nanofluid flow over a vertical plate is considered by Reddy [16]

By considering all the above facts and as suggested in Buongiorno model the two critical slip mechanisms Brownian motion and thermophoresis effects have been carried out in this boundary layer stagnation flow over a non-isothermal stretching sheet through moving water based nanofluid in the presence of suction/injection and constant magnetic flux density.

Gyarmati's variational principle has been employed and the results are presented for temperature profile, concentration profile, the coefficient of skin friction (shear stress), the local Nusselt number (heat transfer) and the local Sherwood number (mass transfer) for various values of velocity ratio parameter $\varepsilon$, suction/injection parameter $H$, magnetic parameter $\xi$, Prandtl number $P r$, wall temperature parameter $n$, Lewis number $L e$ and the slip parameters $N b$ (Brownian effect), $N t$ (thermophoresis effect). The present results are compared with numerical results, and the comparison shows in good agreement. The intention of this study is to establish the fact that Gyarmati's variational principle is one of the exact and most general variational techniques in solving boundary layer flow, heat and mass transfer problems. 


\section{THE GOVERNING BOUNDARY EQUATIONS OF THE SYSTEM}

LAYER

The stretching sheet is assumed to lie along the $x$-axis and infinitely extended downstream with $y$-axis normal to it. The stagnation point of the sheet is at $x=y=0$. The free stream impinges normal to the stretching sheet with velocity $U_{\infty}$ and flows away in the $x$-direction. The sheet stretched with velocity $U_{0}$ by applying two equal and opposite forces along the $x$-axis at the stagnation point as shown in Figure 1. In this study, it is assumed that stretching sheet velocity $U_{0}$ and ambient free stream velocity $U_{\infty}$ are proportional to the distance from the stagnation point. That is, $U_{0}=a x$ and $U_{\infty}=b x$, where $a$ and $b$ are non-zero positive constants.



Figure 1. Schematic diagram

The non-isothermal sheet temperature $T_{0}$ is greater than the ambient temperature $T_{\infty}$. The concentration of the nanofluid near the stretching sheet $C_{0}$ and ambient concentration $C_{\infty}$ are considered as constants.

A uniform magnetic field of strength $B_{0}$ is applied normal to the $x$-axis and assumed that the induced magnetic field, the imposed electric field intensity and the electric field due to the polarization of charges are negligible.

By Oberbeck-Boussinesq boundary layer approximations, the momentum equation has been linearized by neglecting the terms proportional to the product of concentration $(C)$ and temperature $(T)$. This assumption is valid in the case of small temperature gradients in a dilute suspension of nanoparticles.

Hence, the continuity, momentum, thermal and concentration boundary layer equations for steady, two dimensional, incompressible, laminar stagnation point flow of a nanofluid towards a non-isothermal permeable stretching sheet are

$$
\frac{\partial u}{\partial x}+\frac{\partial v}{\partial y}=0
$$

$u \frac{\partial u}{\partial x}+v \frac{\partial u}{\partial y}=U_{\infty} \frac{\partial U_{\infty}}{\partial x}+v \frac{\partial^{2} u}{\partial y^{2}}+\frac{\kappa B_{0}^{2}}{\rho_{f}}\left(U_{\infty}-u\right)$

$$
u \frac{\partial T}{\partial x}+v \frac{\partial T}{\partial y}=\alpha \frac{\partial^{2} T}{\partial y^{2}}+\tau\left\{D_{B}\left(\frac{\partial C}{\partial y} \frac{\partial T}{\partial y}\right)+\frac{D_{B}}{T_{\infty}}\left(\frac{\partial T}{\partial y}\right)^{2}\right\}
$$

$u \frac{\partial C}{\partial x}+v \frac{\partial C}{\partial y}=D_{B} \frac{\partial^{2} C}{\partial y^{2}}+\frac{D_{B}}{T_{\infty}}\left(\frac{\partial^{2} T}{\partial y^{2}}\right)$

subject to the boundary conditions

$$
\begin{aligned}
& y=0 \Rightarrow u=U_{0}=a x, v=v_{0}, T=T_{0}=T_{\infty}+A x^{n}, C=C_{0} \\
& y \rightarrow \infty \Rightarrow u=U_{\infty}=b x, v=0, T=T_{\infty}, C=C_{\infty}
\end{aligned}
$$

Here $u, v, v_{0}, T, C$ are the velocity of the fluid in the $x$ direction, the velocity of the fluid in the $y$-direction, suction/injection speed, the temperature of the nanofluid and concentration of the nanofluid respectively, and $A$ is a constant. The symbols $\mathrm{v}, \kappa, B_{0}, \rho_{f}, \alpha, \tau, D_{B}$ and $D_{T}$ are respectively kinematic viscosity, electric conductivity, externally imposed magnetic field in the $y$-direction, density and thermal diffusivity of the fluid, $\tau=(\rho c)_{\mathrm{p}} /(\rho \mathrm{c})_{\mathrm{f}}$ is the ratio of nanoparticle heat capacity and base fluid heat capacity, Brownian diffusion coefficient and thermophoresis diffusion coefficient.

\section{VARIATIONAL FORMULATION}

By irreversible thermodynamics, Gyarmati [17, 18] developed a variational principle known as "Governing Principle of Dissipative Processes" (GPDP) which is given in its universal form

$\delta \int_{V}(\sigma-\Psi-\Phi) d V=0$

The principle (6) describes the evaluation of linear, quasi linear and some nonlinear irreversible processes at any instant of time and space under constraints that the balance equations

$\rho \dot{a}_{i}+\nabla \cdot \boldsymbol{J}_{i}=\sigma_{i},(i=1,2,3, \cdots, f)$

are satisfied. In Eq. (6), $\delta$ is the variational symbol, $\sigma$ is the entropy production, $\Psi$ and $\Phi$ are dissipation potentials and $V$ is the total volume of the thermodynamic system. In Eq. (7), $\rho$ is the mass density and $\dot{a}_{i}, \boldsymbol{J}_{i}, \sigma_{i}$ are respectively substantial variation, flux and source density of the $i^{\text {th }}$ extensive transport quantity $a_{i}$. The entropy production $\sigma$ per unit volume and unit time can always be written in the bi-linear form

$\sigma=\sum_{i=1}^{f} \boldsymbol{J}_{i} \cdot \boldsymbol{X}_{i} \geq 0$

where $\boldsymbol{J}_{\boldsymbol{i}}$ and $\boldsymbol{X}_{\boldsymbol{i}}$ are fluxes and forces respectively. According to Onsager's linear theory $[19,20]$, the fluxes are linear functions of forces, that is

$\boldsymbol{J}_{i}=\sum_{k=1}^{f} L_{i k} \cdot \boldsymbol{X}_{k}, \quad(i=1,2,3, \cdots, f)$

or alternatively

$\boldsymbol{X}_{i}=\sum_{k=1}^{f} R_{i k} \cdot \boldsymbol{J}_{k}, \quad(i=1,2,3, \cdots, f)$. 
The constants $L_{i k}$ and $R_{i k}$ are conductivities and resistances respectively, satisfying the reciprocal relations $[19,20]$.

$L_{i k}=L_{k i}$ and $R_{i k}=R_{k i} \quad(i, k=1,2,3, \cdots, f)$

The matrices of $L_{i k}$ and $R_{i k}$ are mutually reciprocals, and they are symmetric, that is

$$
\sum_{m=1}^{f} L_{i m} R_{m k}=\sum_{m=1}^{f} L_{m k} R_{i m}=\delta_{i k},(i, k=1,2,3, \cdots, f)
$$

where $\delta_{i k}$ is the Kronecker delta.

The local dissipation potentials $\Psi$ and $\Phi$ are defined [19, 20] as,

$\Psi(\boldsymbol{X}, \boldsymbol{X})=\left(\frac{1}{2}\right) \sum_{i, k=1}^{f} L_{i k} \boldsymbol{X}_{i} \cdot \boldsymbol{X}_{k} \geq 0$

$\Phi(\boldsymbol{J}, \boldsymbol{J})=\left(\frac{1}{2}\right) \sum_{i, k=1}^{f} R_{i k} \boldsymbol{J}_{i} \cdot \boldsymbol{J}_{k} \geq 0$.

In the case of transport processes, the forces $\boldsymbol{X}_{i}$ can be generated as gradients of certain " $\Gamma$ " variables and can be written as

$\boldsymbol{X}_{i}=\nabla \Gamma_{i}$

The principle (6) with the help of Eqs (8), (13), (14) and (15), takes the form

$$
\begin{array}{r}
\delta \int_{V}\left[\sum_{i=1}^{f} \boldsymbol{J}_{i} \cdot \nabla \Gamma_{i}-\left(\frac{1}{2}\right) \sum_{i, k=1}^{f} L_{i k} \nabla \Gamma_{i} \cdot \nabla \Gamma_{k}\right. \\
\left.-\left(\frac{1}{2}\right) \sum_{i, k=1}^{f} R_{i k} \boldsymbol{J}_{i} \cdot \boldsymbol{J}_{k}\right] d V=0
\end{array}
$$

This variational principle has been already applied for various dissipative systems and was established as the most general and exact variational principle of macroscopic continuum physics. Many other variational principles have already been shown as partial forms of Gyarmati's principle.

The balance equations of the system play a central role in the formulation of Gyarmati's variational principle and hence the governing boundary layer Equations (1 - 4) are written in the balance form as

$\nabla \cdot \boldsymbol{V}=0, \quad(\boldsymbol{V}=u \boldsymbol{i}+v \boldsymbol{j})$

$\rho_{f}(\boldsymbol{V} \cdot \nabla) \boldsymbol{V}+\nabla \cdot \overline{\bar{P}}=\left(\kappa B_{0}^{2}\right)\left[U_{\infty}-(\boldsymbol{i} \cdot \boldsymbol{V})\right]$

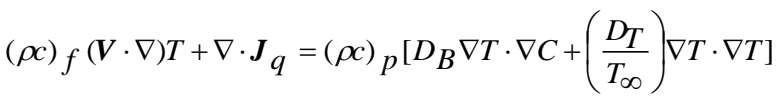

$\rho_{p}(\boldsymbol{V} \cdot \nabla) C+\nabla \cdot \boldsymbol{J}_{C}=\rho_{p}\left(\frac{D_{T}}{T_{\infty}}\right) \nabla^{2} T$

These equations represent the mass, momentum, energy and concentration balances respectively. Here $\boldsymbol{i}$ and $\boldsymbol{j}$ being unit vectors in the directions of $x$ and $y$ axes respectively. In Eq (18) $\overline{\bar{P}}$ denotes the pressure tensor which can be decomposed [17] as

$\overline{\bar{P}}=p \overline{\bar{\delta}}+\overline{\bar{P}}^{v} v s$

where $p$ is the hydrostatic pressure, $\overline{\bar{\delta}}$ is the unit tensor and $\frac{o}{\bar{P}} v s$ pressure tensor of trace zero. In Eq (19) $\boldsymbol{J}_{q}$ denotes heat current density which can be written as $\boldsymbol{J}_{q}=-L_{\lambda} \nabla T$. In Eq (20) $\boldsymbol{J}_{c}$ represents mass current density and is defined as $\boldsymbol{J}_{c}=-L_{c} \nabla C$.

In the study of thermo hydrodynamics problems, the energy picture of Governing Principle of Dissipative Processes is always advantageous over entropy picture. Therefore, the energy dissipation $T \sigma$ is used instead of entropy production $\sigma$. The energy dissipation for the present system is given [17] by,

$T \sigma=-P_{12}\left(\frac{\partial u}{\partial y}\right)-J_{q}\left(\frac{\partial \ln T}{\partial y}\right)-J_{c}\left(\frac{\partial C}{\partial y}\right)$

In energy picture, the actual variable is $\ln T$ instead of $T$.

Here $P_{12}$ is the only component of momentum flux $\frac{o}{\bar{P}} v s, J_{q}$ is the heat flux, and $J_{c}$ is the mass flux, satisfy the constitutive relations connecting the independent fluxes and forces as

$$
\begin{aligned}
& P_{12}=-L_{s}\left(\frac{\partial u}{\partial y}\right) \\
& J_{q}=-L_{\lambda}\left(\frac{\partial \ln T}{\partial y}\right) \\
& J_{c}=-L_{c}\left(\frac{\partial C}{\partial y}\right)
\end{aligned}
$$

where $L_{s}=\mu, L_{\lambda}=\lambda T$ and $L_{c}=\rho_{p} D_{B}$, where $\mu, \lambda$ and $D_{B}$ are viscosity, thermal conductivity and partial diffusion coefficient respectively. With the help of Eq (23), the dissipation potentials in energy picture are found as follows

$$
\begin{aligned}
& T \Psi=\left(\frac{1}{2}\right)\left[L_{S}\left(\frac{\partial u}{\partial y}\right)^{2}+L_{\lambda}\left(\frac{\partial \ln T}{\partial y}\right)^{2}+L_{c}\left(\frac{\partial C}{\partial y}\right)^{2}\right] \\
& T \Phi=\left(\frac{1}{2}\right)\left[R_{S} P_{12}^{2}+R_{\lambda} J_{q}^{2}+R_{C} J_{c}^{2}\right]
\end{aligned}
$$

where $L_{S}=R_{S}^{-1}, L_{\lambda}=R_{\lambda}^{-1}$ and $L_{c}=R_{c}^{-1}$.

Using Equations (22 - 25), Gyarmati's variational principle (6) in energy picture is formulated in the following form

$$
\begin{aligned}
\delta \int_{0}^{l} \int_{0}^{\infty}[- & P_{12}\left(\frac{\partial u}{\partial y}\right)-J_{q}\left(\frac{\partial \ln T}{\partial y}\right)-J_{C}\left(\frac{\partial C}{\partial y}\right) \\
& -\frac{L_{s}}{2}\left(\frac{\partial u}{\partial y}\right)^{2}-\frac{L_{\lambda}}{2}\left(\frac{\partial \ln T}{\partial y}\right)^{2}-\frac{L_{c}}{2}\left(\frac{\partial C}{\partial y}\right)^{2} \\
& \left.-\left(\frac{R_{s}}{2}\right) P_{12}^{2}-\left(\frac{R_{\lambda}}{2}\right) J_{q}^{2}-\left(\frac{R_{c}}{2}\right) J_{c}^{2}\right] d y d x=0,
\end{aligned}
$$


in which $l$ is the typical length of the surface.

\section{METHOD OF SOLUTION}

It is assumed that the trial functions for velocity, temperature and concentration fields inside the respective boundary layers are as follows

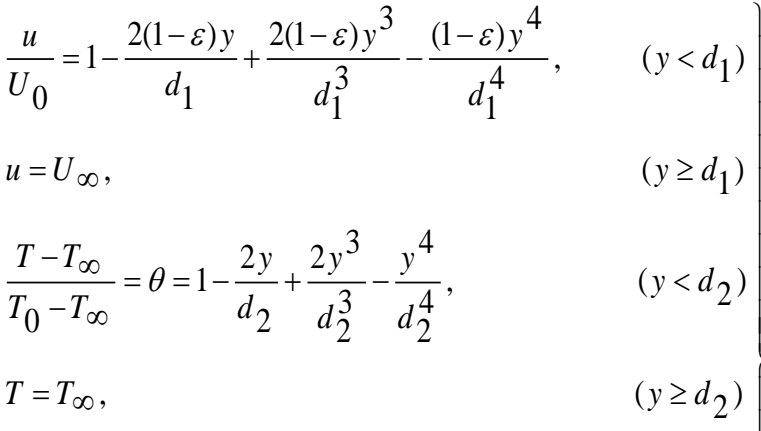

$$
\begin{aligned}
& \frac{C-C_{\infty}}{C_{0}-C_{\infty}}=\phi=1-\frac{3 y}{d_{3}}+\frac{3 y^{2}}{d_{3}^{2}}-\frac{y^{3}}{d_{3}^{3}}, \quad\left(y<d_{3}\right) \\
& C=C_{\infty}, \quad\left(y \geq d_{3}\right)
\end{aligned}
$$

where $d_{1}, d_{2}, d_{3}$ are the velocity, temperature and concentration boundary layer thicknesses which are to be determined from the variational procedure, and $\varepsilon\left(=\mathrm{U}_{\infty} / \mathrm{U}_{0}\right)$ is the velocity ratio.

The trial functions (27) satisfy the following boundary conditions,

$$
\begin{aligned}
& y=0 \Rightarrow u=U_{0}=a x, v=v_{0}, T=T_{0}=T_{\infty}+A x^{n}, \\
& C=C_{0}, \frac{\partial^{2} u}{\partial y^{2}}=\frac{\partial^{2} T}{\partial y^{2}}=\frac{\partial^{2} C}{\partial y^{2}}=0 \\
& y=d_{1} \Rightarrow u=U_{\infty}=b x, \frac{\partial u}{\partial y}=0, \frac{\partial^{2} u}{\partial y^{2}}=0 \\
& y=d_{2} \Rightarrow T=T_{\infty}, \frac{\partial T}{\partial y}=0, \frac{\partial^{2} T}{\partial y^{2}}=0 \\
& y=d_{3} \Rightarrow C=C_{\infty}, \frac{\partial C}{\partial y}=0, \frac{\partial^{2} C}{\partial y^{2}}=0
\end{aligned}
$$

The smooth fit conditions $\frac{\partial u}{\partial y}=0, \frac{\partial T}{\partial y}=0$ and $\frac{\partial C}{\partial y}=0$ correspond to $P_{12}=0, J_{q}=0$ and $J_{c}=0$ at their respective edges of the boundary layer. The curvatures $\left(\frac{\partial^{2} u}{\partial y^{2}}, \frac{\partial^{2} T}{\partial y^{2}}, \frac{\partial^{2} C}{\partial y^{2}}\right)$ of the velocity, temperature and concentration profiles are negligibly small at the wall due to no pressure gradient and turn large in the immediate neighborhood of the wall, then asymptotically decay to zero so that the curvatures are continuous at their respective edges of the boundary layer. Using the boundary conditions (28), the transverse velocity component $v$ is obtained from the mass balance equation (17) as

$$
v=\frac{a(1-\varepsilon) y^{5}}{5 d_{1}^{4}}-\frac{a(1-\varepsilon) y^{4}}{2 d_{1}^{3}}+\frac{a(1-\varepsilon) y^{2}}{d_{1}}-a y+v_{0}
$$

The velocity, temperature and concentration functions (27) and the boundary conditions (28) are used in the governing boundary layer equations (17 - 20). And on direct integration on $y$ with the help of their corresponding smooth fit conditions $u_{y}=0, T_{y}=0$ and $C_{y}=0$, the momentum flux $P_{12}$, energy flux $J_{q}$ and mass flux $J_{c}$ are obtained with the assumption that Prandtl number $\operatorname{Pr} \geq 1$, that is $d_{1} \geq d_{2}$.

Using the expressions of $P_{12}, J_{q}$, and $J_{c}$ together with the velocity, temperature and concentration functions (27), the variational principle (26) is formulated. After performing the integration on $y$, one can obtain the variational principle in the following forms,

$$
\delta \int_{0}^{l} L\left[d_{1}, d_{2}, d_{3}, d_{1}^{\prime}, d_{2}^{\prime}, d_{3}^{\prime}\right] d x=0, \quad(1 \leq \operatorname{Pr} \leq \infty)
$$

where $L$ is the Lagrangian density of the principle. The prime indicates differentiation with respect to $x$. The variation is carried out on the independent parameters $d_{1}, d_{2}$ and $d_{3}$. The Euler-Lagrange equations corresponding to this variational principle are

$$
\begin{aligned}
& \frac{\partial L}{\partial d_{1}}-\frac{d}{d x}\left(\frac{\partial L}{\partial d_{1}^{\prime}}\right)=0 \\
& \frac{\partial L}{\partial d_{2}}-\frac{d}{d x}\left(\frac{\partial L}{\partial d_{2}^{\prime}}\right)=0, \quad(1 \leq \mathbf{P r} \leq \infty)
\end{aligned}
$$

$\frac{\partial L}{\partial d_{3}}-\frac{d}{d x}\left(\frac{\partial L}{\partial d_{3}^{\prime}}\right)=0$

These Eqs (31) - (33) are second order ordinary differential equations in terms of $d_{1}, d_{2}$ and $d_{3}$.

The procedure for solving Eqs (31), (32) and (33) can be considerably simplified by introducing the non-dimensional boundary layer thicknesses $d_{1}{ }^{*}, d_{2}{ }^{*}$ and $d_{3}{ }^{*}$ which are given by

$$
\left.\begin{array}{l}
d_{1}=d_{1}^{*} \sqrt{\frac{v x}{U_{\mathrm{O}}}} \\
d_{2}=d_{2}^{*} \sqrt{\frac{v x}{U_{\mathrm{O}}}} \\
\text { and } \\
d_{3}=d_{3}^{*} \sqrt{\frac{v x}{U_{\mathrm{O}}}}
\end{array}\right\}
$$

The variational principle (30) is subject to the transformations (34) and the resulting Euler-Lagrange equations are obtained as simple polynomial equations,

$$
\frac{\partial L}{\partial d_{1}^{*}}=0
$$


$\frac{\partial L}{\partial d_{2}^{*}}=0, \quad(1 \leq \operatorname{Pr} \leq \infty)$

$\frac{\partial L}{\partial d_{3}^{*}}=0$

The coefficients of these Eqs (35), (36) and (37) dependent on the independent parameters $\varepsilon, H, \xi, P r, n, L e, N b$ and $N t$, where $\varepsilon=\frac{U_{\infty}}{U_{0}}$ (velocity ratio parameter), $H=v_{0} \sqrt{\frac{x}{v_{f} U_{0}}}$ (suction/injection parameter), $\quad \xi=v_{0} \sqrt{\frac{\kappa B_{0}^{2}}{\rho U_{0}}} \quad$ (magnetic parameter), $\operatorname{Pr}=\frac{v_{f}}{\alpha_{f}}$ (Prandtl number), wall temperature parameter $\quad n, \quad L e=\frac{v}{D_{B}} \quad$ (Lewis number), $N b=\frac{\tau D_{B}\left(C_{0}-C_{\infty}\right)}{v} \quad$ (Brownian motion parameter) and $N t=\frac{\tau D_{T}\left(T_{0}-T_{\infty}\right)}{v T_{\infty}}$ (thermophoresis parameter).

In the present analysis, the suction and injection are represented respectively, by $H<0$ and $H>0$. Eqs (35), (36) and (37) are simple coupled polynomial equations and it can be solved for any values of $\varepsilon, H, \xi, P r, n, L e, N b$ and $N t$ and it is obtained as simultaneous solution of $d_{1}{ }^{*}, d_{2}{ }^{*}$ and $d_{3}{ }^{*}$. After getting the values of $d_{1}{ }^{*}, d_{2}{ }^{*}$ and $d_{3}{ }^{*}$ for given $\varepsilon, H, \xi, P r, n$, $\mathrm{Le}, \mathrm{Nb}$ and $\mathrm{Nt}$, the values of temperature, concentration profiles, skin friction (shear stress), heat transfer (Nusselt number) and mass transfer (Sherwood number) are calculated with the help of the following expressions,

$\eta=y \sqrt{\frac{U_{0}}{v x}}$

$\tau_{w}=\sqrt{\frac{v x}{U_{0}^{3}}}\left(\frac{-P_{12}}{L_{s}}\right)_{y=0}$

$N u_{l}=\sqrt{\frac{v x}{U_{0}\left(T_{0}-T_{\infty}\right)^{2}}}\left(\frac{J_{q}}{L_{\lambda}}\right)_{y=0}$

and $S h_{l}=\sqrt{\frac{v x}{U_{0}\left(C_{0}-C_{\infty}\right)^{2}}}\left(\frac{J_{c}}{L_{c}}\right)_{y=0}$

In terms of non-dimensional parameters, the dimensionless forms of skin friction and rate of heat and mass transfer expressions are respectively obtained as,

$\tau_{w}=479 d_{1}{ }^{*} / 1800-7 H / 10+91 \varepsilon d_{1}{ }^{*} / 900+7 \xi d_{1}{ }^{*} / 30-\varepsilon / d_{1}{ }^{*}$

- $661 \varepsilon^{2} d_{1}{ }^{*} / 1800+1 / d_{1}{ }^{*}+7 \varepsilon H / 10-7 \varepsilon \xi d_{l}{ }^{*} / 30$

$\mathrm{Nu}_{l}=3 \operatorname{Prd}_{2}{ }^{*} / 10-\operatorname{PrH}+3 \operatorname{Prd}_{2}{ }^{*} n / 10-2 \operatorname{PrNt} / d_{2}{ }^{*}$
$+6 \operatorname{PrNtd}_{3}{ }^{*} / d_{2}{ }^{* 3}-4 \operatorname{PrNtd}{ }_{3}{ }^{*} / d_{2}{ }^{* 4}+\mathrm{LePrHNb}$

- $\operatorname{LePrNbd}_{3}{ }^{*} 4+2 \operatorname{Prd}_{2}{ }^{{ }_{2}}(\varepsilon-1) / 15 d_{1}{ }^{*}-3 \operatorname{Prd}_{2}{ }^{* 4}(\varepsilon-1) / 140 d_{1}{ }^{* 3}$

$+\operatorname{Prd}_{2}{ }^{* 5}(\varepsilon-1) / 180 d_{1}{ }^{* 4}+2 \operatorname{Prd}_{2}{ }^{* 2} n(\varepsilon-1) / 15 d_{1}{ }^{*}$

- $3 \operatorname{Prd}_{2}{ }^{* 4} n(\varepsilon-1) / 140 d_{1}{ }^{* 3}+\operatorname{Prd}_{2}{ }^{* 5} n(\varepsilon-1) / 180 d_{1}{ }^{* 4}$

$+\operatorname{LePrNbd}_{2}{ }^{* 2} / 5 d_{3}{ }^{*}-\operatorname{LePrNbd}_{2}{ }^{* 3} / 7 d_{3}{ }^{* 2}$

$+9 \operatorname{LePrNbd}_{2}{ }^{* 4} / 280 d_{3}{ }^{* 3}+2 \mathrm{LePrHNbd}_{2}{ }^{{ }_{2}} / 5 \mathrm{~d}_{3}{ }^{{ }^{2} 2}$

- $\mathrm{LePrHNbd}_{2}{ }^{* 3} / 14 d_{3}{ }^{* 3}-\operatorname{LePrNbd}_{3}{ }^{* 2}(\varepsilon-1) / 10 d_{1}{ }^{*}$

$+\operatorname{LePrNbd}_{3}{ }^{* 4}(\varepsilon-1) / 70 d_{1}{ }^{* 3}-\operatorname{LePrNbd}_{3}{ }^{*}(\varepsilon-1) / 280 d_{1}{ }^{* 4}$

- $9 \operatorname{LePrHNbd}_{2}{ }^{*} 10 d_{3}{ }^{*}+\operatorname{LePrNbd}_{2}{ }^{*}{ }^{3}(\varepsilon-1) / 14 d_{1}{ }^{*} d_{3}{ }^{*}$

- $9 \operatorname{LePrNbd}_{2}{ }^{* 4}(\varepsilon-1) / 140 d_{1}{ }^{*} d_{3}{ }^{* 2}$

$+\operatorname{LePrNbd}_{2}{ }^{* 5}(\varepsilon-1) / 60 d_{1}{ }^{*} d 3^{* 3}$

- $\operatorname{LePrNbd}_{2}{ }^{* 5}(\varepsilon-1) / 120 d_{1}{ }^{* 3} d_{3}{ }^{*}$

$+\operatorname{LePrNbd}_{2}{ }^{* 6}(\varepsilon-1) / 105 d_{1}{ }^{* 3} d_{3}{ }^{{ }^{2}}$

$+\operatorname{LePrNbd}_{2}{ }^{* 6}(\varepsilon-1) / 525 d_{1}{ }^{* 4} d_{3}{ }^{*}$

- $9 \operatorname{LePrNb}_{2}{ }^{* 7}(\varepsilon-1) / 3080 d_{1}{ }^{* 3} d_{3}{ }^{* 3}$

- $9 \operatorname{LePrNbd}_{2}{ }^{{ }^{*}}(\varepsilon-1) / 3850 d_{1}{ }^{* 4} d_{3}{ }^{* 2}$

$+\operatorname{LePrNbd}_{2}{ }^{* 8}(\varepsilon-1) / 1320 d_{1}{ }^{* 4} d_{3}{ }^{* 3}$

$\mathrm{Sh}_{l}=\mathrm{Led}_{3}{ }^{*} / 4-\mathrm{LeH}+2 \mathrm{PrNt} / \mathrm{d}_{2}{ }^{*}-3 \mathrm{PrNtd}_{3}{ }^{{ }^{*}} / 5 \mathrm{~d}_{2}{ }^{* 3}$

$+\operatorname{PrNtd}_{3}{ }^{* 3} / 5 d_{2}{ }^{* 4}+\operatorname{Led}_{3}{ }^{* 2}(\varepsilon-1) / 10 d_{1}{ }^{*}$

- $\operatorname{Led}_{3}{ }^{* 4}(\varepsilon-1) / 70 d_{1}{ }^{*}+\operatorname{Led}_{3}{ }^{* 5}(\varepsilon-1) / 280 d_{1}{ }^{* 4}$

- $\operatorname{PrNtd}_{3}{ }^{{ }^{2}} / \mathrm{Nbd}_{2}{ }^{*}+4 \mathrm{PrNt}^{2} \mathrm{~d}_{3}{ }^{*} / \mathrm{Nbd}_{2}{ }^{{ }_{2}}$

$+3 \mathrm{PrNtd}_{3}{ }^{* 4} / 2 \mathrm{Nbd}_{2}{ }^{* 3}-4 \mathrm{PrNtd}_{3}{ }^{*} / 5 \mathrm{Nbd}_{2}{ }^{*}$

- $\operatorname{PrNtd}_{3}{ }^{*} n / \mathrm{Nb}-8 \mathrm{PrNt}^{2} \mathrm{~d}_{3}{ }^{{ }_{3}} / \mathrm{Nbd}_{2}{ }^{{ }_{4}}+4 \mathrm{PrNt}^{2} \mathrm{~d}_{3}{ }^{{ }_{4}} / \mathrm{Nbd}_{2}{ }^{{ }_{5}}$

$+36 \operatorname{PrNt}^{2} \mathrm{~d}_{3}{ }^{*} / 5 \mathrm{Nbd}_{2}{ }^{{ }^{6} 6}-8 \mathrm{PrNt}^{2} \mathrm{~d}_{3}{ }^{* 6} / \mathrm{Nbd}_{2}{ }^{* 7}$

$+16 \mathrm{PrNt}^{2} \mathrm{~d}_{3}{ }^{* 7} / 7 \mathrm{Nbd}_{2}{ }^{* 8}+2 \mathrm{PrHNt}_{3}{ }^{*} / \mathrm{Nbd}_{2}{ }^{*}$

- $2 \mathrm{PrHNtd}_{3}{ }^{*} / \mathrm{Nbd}_{2}{ }^{* 3}+\mathrm{PrHNtd}_{3}{ }^{* 4} / \mathrm{Nbd}_{2}{ }^{* 4}$

$+\operatorname{PrNtd}_{3}{ }^{* 2} n / \mathrm{Nbd}_{2}{ }^{*}-\operatorname{PrNtd}_{3}{ }^{*}{ }^{4} n / 2 \mathrm{Nbd}_{2}{ }^{* 3}$

$+\operatorname{PrNtd}_{3}{ }^{* 5} n / 5 \mathrm{Nbd}_{2}{ }^{* 4}-\mathrm{PrNtd}_{3}{ }^{* 2} n(\varepsilon-1) / \mathrm{Nbd}_{1}{ }^{*}$

$+\operatorname{PrNtd}_{3}{ }^{* 4} n(\varepsilon-1) / 2 \mathrm{Nbd}_{1}{ }^{* 3}-\mathrm{PrNtd}_{3}{ }^{* 5} n(\varepsilon-1) / 5 \mathrm{Nbd}_{1}{ }^{* 4}$

- $2 \operatorname{PrNtd}_{3}{ }^{*}(\varepsilon-1) / 3 \mathrm{Nbd}_{1}{ }^{*} d_{2}{ }^{*}+6 \operatorname{PrNtd}_{3}{ }^{* 5}(\varepsilon-1) / 5 \mathrm{Nbd}_{1}{ }^{*} d_{2}{ }^{* 3}$

$+\operatorname{PrNtd}_{3}{ }^{* 5}(\varepsilon-1) / 5 \mathrm{Nbd}_{1}{ }^{* 3}{d_{2}}{ }^{*}-2 \operatorname{PrNtd}_{3}{ }^{* 6}(\varepsilon-1) / 3 \mathrm{Nbd}_{1}{ }^{*} d_{2}{ }^{* 4}$

- $\operatorname{PrNtd}_{3}{ }^{* 6}(\varepsilon-1) / 15 \mathrm{Nbd}_{1}{ }^{* 4} d_{2}{ }^{*}-3 \operatorname{PrNtd}_{3}{ }^{* 7}(\varepsilon-1) / 7 \mathrm{Nbd}_{1}{ }^{* 3}{d_{2}}^{* 3}$

$+\operatorname{PrNtd}_{3}{ }^{* 8}(\varepsilon-1) / 4 \mathrm{Nbd}_{1}{ }^{* 3} \mathrm{~d}_{2}{ }^{* 4}+3 \operatorname{PrNtd}_{3}{ }^{* 8}(\varepsilon-1) / 20 \mathrm{Nbd}_{1}{ }^{* 4} d_{2}{ }^{* 3}$

- $4 \operatorname{PrNtd}_{3}{ }^{* 9}(\varepsilon-1) / 45 \mathrm{Nbd}_{1}{ }^{* 4} \mathrm{~d}_{2}{ }^{* 4}+4 \mathrm{PrNtd}_{3}{ }^{* 3} n(\varepsilon-1) / 3 \mathrm{Nbd}_{1}{ }^{*} d_{2}{ }^{*}$

- $4 \operatorname{PrNtd}_{3}{ }^{* 5} n(\varepsilon-1) / 5 \mathrm{Nbd}_{1}{ }^{*} d_{2}{ }^{* 3}-4 \mathrm{PrNt}_{3}{ }^{* 5} n(\varepsilon-1) / 5 \mathrm{Nbd}_{1}{ }^{* 3} d_{2}{ }^{*}$

$+\operatorname{PrNtd}_{3}{ }^{* 6} n(\varepsilon-1) / 3 \mathrm{Nbd}_{1}{ }^{*} d_{2}{ }^{* 4}+\operatorname{PrNtd}_{3}{ }^{*} n(\varepsilon-1) / 3 \mathrm{Nbd}_{1}{ }^{* 4} d_{2}{ }^{*}$

$+4 \operatorname{PrNt}_{3}{ }^{* 7} n(\varepsilon-1) / 7 \mathrm{Nbd}_{1}{ }^{* 3} d_{2}{ }^{* 3}-\operatorname{PrNtd}_{3}{ }^{*} n(\varepsilon-1) / 4 N b d_{1}{ }^{* 3} d_{2}{ }^{* 4}$

- $\operatorname{PrNtd}_{3}{ }^{* 8} n(\varepsilon-1) / 4 N b d_{1}{ }^{* 4} d_{2}{ }^{* 3}+\operatorname{PrNtd}_{3}{ }^{* 9} n(\varepsilon-1) / 9 N b d_{1}{ }^{* 4} d_{2}{ }^{* 4}$

\section{RESULTS AND DISCUSSION}

To justify the accuracy of the present variational technique, the obtained results are compared with available numerical solutions in the literature.

Table 1. Skin friction for various values of $\varepsilon$ when $\xi=H=0$

\begin{tabular}{cccc}
\hline$\varepsilon$ & Present Results & Ibrahim et al. [13] & $\begin{array}{c}\text { Mahapatra and } \\
\text { Gupta [7] } \\
\tau_{\mathrm{w}}\end{array}$ \\
\hline 0.01 & 1.064118317 & $\tau_{\mathrm{w}}$ & - \\
0.1 & 1.023414638 & 0.9980 & 0.9694 \\
0.2 & 0.962050351 & 0.9694 & 0.9181 \\
0.5 & 0.690246302 & 0.6673 & 0.6673 \\
2.0 & -2.050841819 & -2.0175 & -2.0175 \\
3.0 & -4.791630607 & -4.7292 & -4.7293 \\
\hline
\end{tabular}

Table 1 displays the skin friction values for different values of velocity ratio parameter $\varepsilon$, when $\xi=H=0$. From this table, the comparison of the present skin friction values with the 
results of Ibrahim et al. [13] and Mahapatra and Gupta [7] is in good agreement. And it is observed that skin friction decreases with increase in velocity ratio parameter $\varepsilon$.

Table 2. Local Nusselt number for various values of $\operatorname{Pr}$ and $\varepsilon$ when $H=\xi=n=N b=N t=0$

\begin{tabular}{ccccc}
\hline Pr & $\varepsilon$ & $\begin{array}{c}\text { Present } \\
\text { Results } \\
N u_{l}\end{array}$ & $\begin{array}{c}\text { Ibrahim et } \\
\text { al. [13] } \\
N u_{l}\end{array}$ & $\begin{array}{c}\text { Mahapatra and } \\
\text { Gupta [7] } \\
N u_{l}\end{array}$ \\
\hline 1 & 0.1 & 0.590485143 & 0.6022 & 0.603 \\
& 0.2 & 0.617039895 & 0.6245 & 0.625 \\
& 0.5 & 0.690363927 & 0.6924 & 0.692 \\
1.5 & 0.1 & 0.773217299 & 0.7768 & 0.777 \\
& 0.2 & 0.795416232 & 0.7971 & 0.797 \\
& 0.5 & 0.864975099 & 0.8648 & 0.863 \\
\hline
\end{tabular}

Table 2 exhibits the heat transfer values of conventional fluid for various values of velocity ratio parameter $\varepsilon$, and Prandtl number $\operatorname{Pr}$, when $\xi=H=n=0$. From this table, it is evidently clear that the comparison of the present heat transfer rates obtained by the present variational technique with other numerical methods by Ibrahim et al. [13] and Mahapatra and Gupta [7] is excellent with a higher order of accuracy.

Further, Prasad and Vajravelu [21] gave the numerical solution for non-dimensional skin friction value as 1.00029111 in the absence of magnetic field for impermeable stretching sheet whereas the computed present value is 1.067727442 when $\varepsilon=\xi=H=0$.

In this study, the physical quantities of practical interest skin friction, temperature, concentration profiles, heat and mass transfer rates are obtained for the cases of suction and injection and these cases are compared with the impermeable case. Further, the investigation has been carried out for water based nanofluid at a fixed Prandtl number, $P r=6.2$.

Figure 2, presents the effects of shear stress as a function of $\xi$ for various values of velocity ratio parameter $\varepsilon$. From these illustrations, it is observed that the skin friction is an increasing function of $\xi$ for $\varepsilon<1$ but it decreases with the increasing values of $\xi$ when $\varepsilon>1$ while the values of skin friction independent upon $\xi$ when $\varepsilon=1$. Also, it is observed that values of skin friction are negative when $\varepsilon>1$ due to the drag force exerted by the fluid of high free stream velocity compared to sheet velocity. Further, it is observed that suction increases and injection decreases the absolute value of skin friction when compared to the impermeable sheet.

In Figure 3, the influence of diffusion parameters on temperature profile for suction, injection, and impermeable cases are presented. From these illustrations, it is clear that the temperature is an increasing function of Brownian motion and thermophoresis parameters. Since these diffusion parameters increase the thermal boundary layer thickness, the temperature profile is fuller for higher values of $N b$ and $N t$. It is observed from these figures that the temperature decreases rapidly in the case of suction because the suction decreases the boundary layer thickness and hence increases the heat transfer.

Figure 4 illustrates the variation of temperature profile on velocity ratio parameter $\varepsilon$ for permeable and impermeable cases. The observation depicts that the temperature distribution increases as velocity ratio parameter $\varepsilon$ decreases and this increase is not at a significant level when suction controls the boundary layer. Also, it is noted that when $\mathrm{U}_{\infty}$ $>\mathrm{U}_{0}$, the temperature profile rapidly attains the ambient temperature.
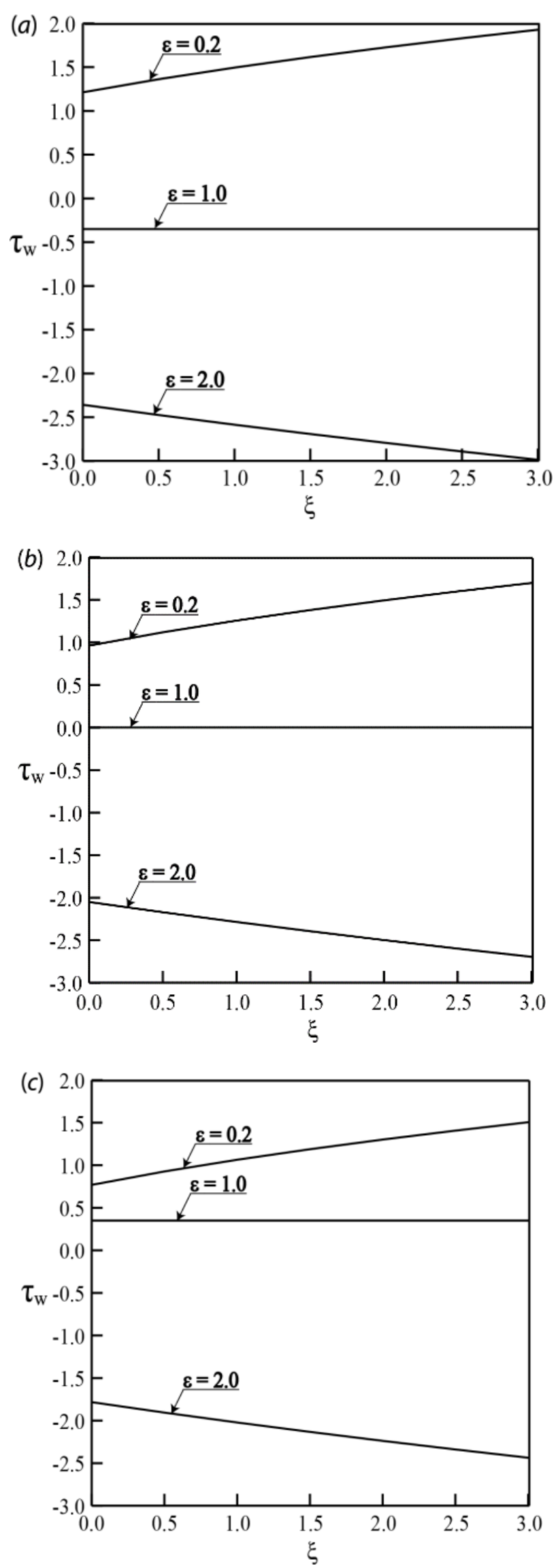

Figure 2. Skin friction values as a function of $\xi$ for different values of $\varepsilon$ when (a) $H=-0.5$ (suction), (b) $H=0.0$ and (c) $H=0.5$ (injection) 

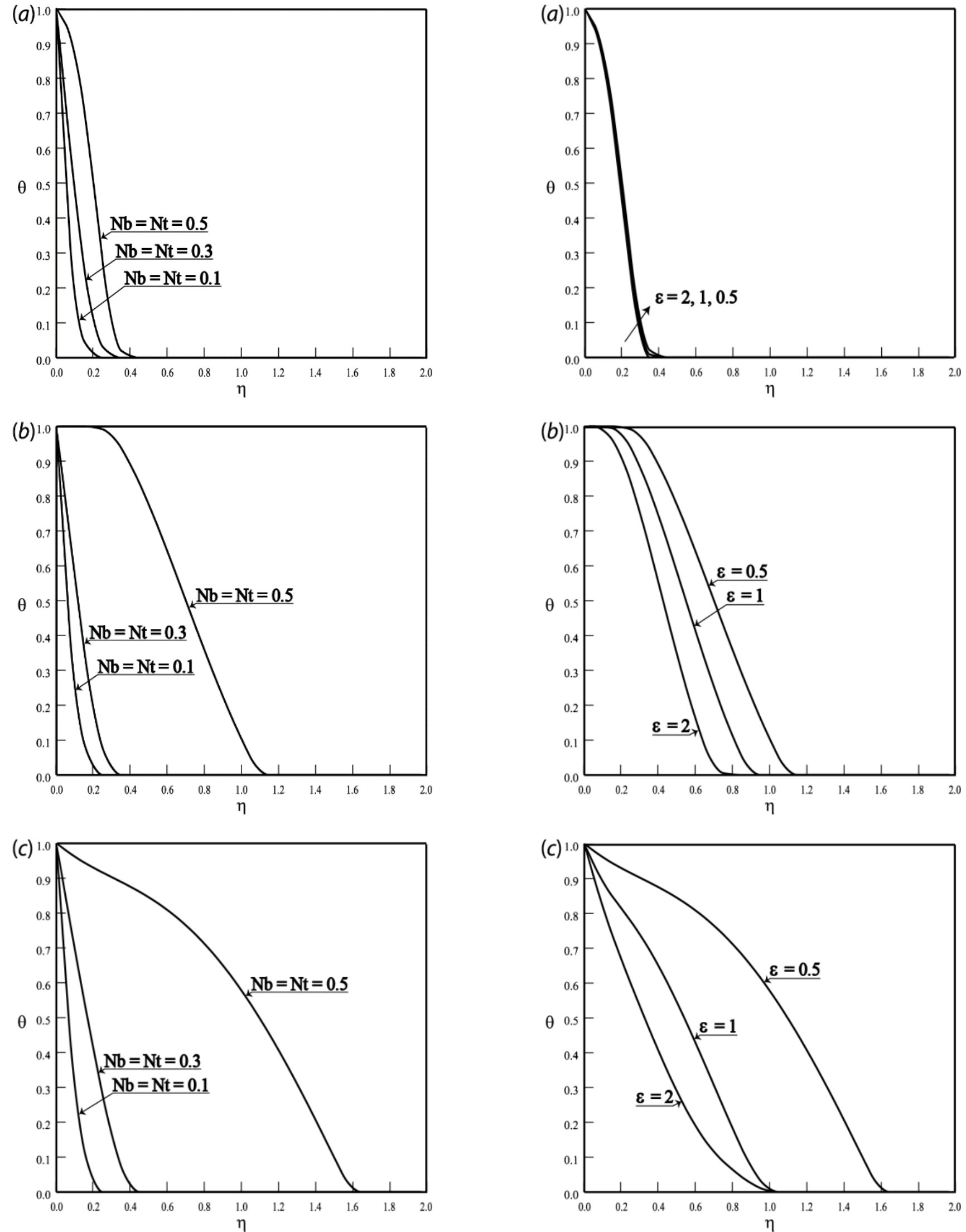

Figure 3. Temperature profile for $\operatorname{Pr}=6.2, L e=10, n=\xi=1$, $\varepsilon=0.5$ and $N b=N t=0.1,0.3,0.5$ when $(a) H=-0.5$ (suction), (b) $H=0.0$ and (c) $H=0.5$ (injection)

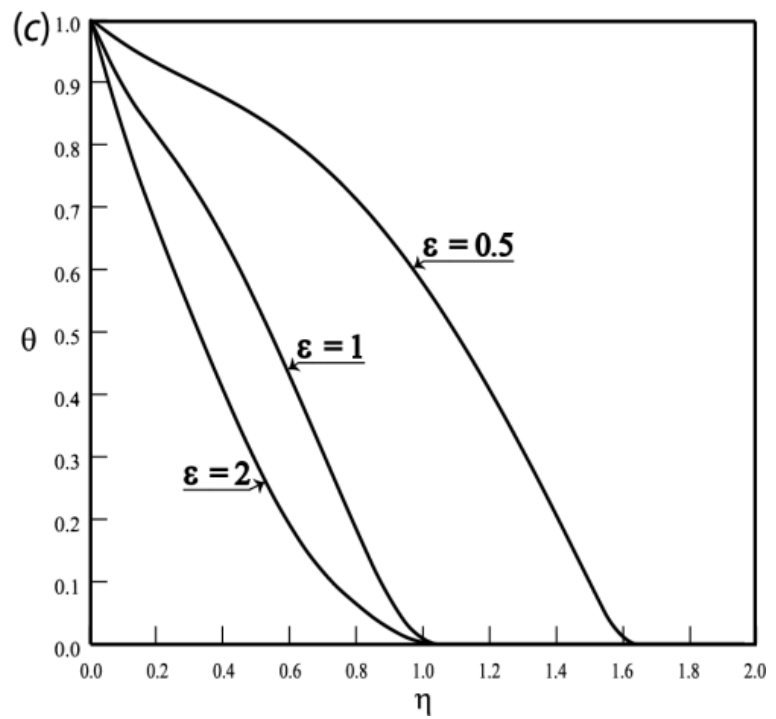

Figure 4. Temperature profile for $\operatorname{Pr}=6.2, L e=10, n=\xi=1$, $N b=N t=0.5$ and $\varepsilon=0.5,1,2$ when (a) $H=-0.5$ (suction), (b) $H=0.0$ and (c) $H=0.5$ (injection) 

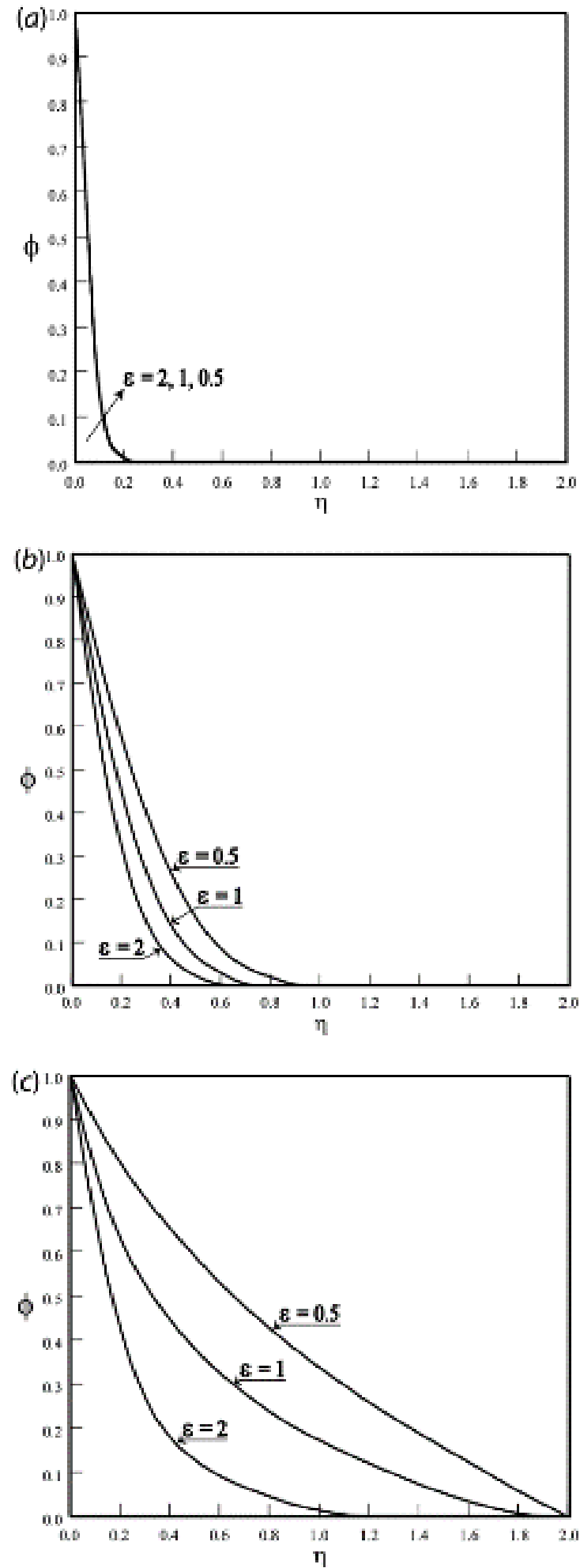

Figure 5. Concentration profile for $\operatorname{Pr}=6.2, L e=10, n=\xi=1$, $N b=N t=0.5$ and $\varepsilon=0.5,1,2$ when $(a) H=-0.5$ (suction), (b) $H=0.0$ and (c) $H=0.5$ (injection)

Figure 5 demonstrates the effects of velocity ratio parameter $\varepsilon$ on concentration distribution. Increasing values of $\varepsilon$ decreases the concentration profile due to the fact that as $\varepsilon$ increases the concentration boundary layer thickness decreases. Also, it is evidently clear that, since suction decreases and injection increase the concentration boundary layer thickness, concentration profile are decreasing and increasing respectively for suction and injection when compared to the impermeable case.
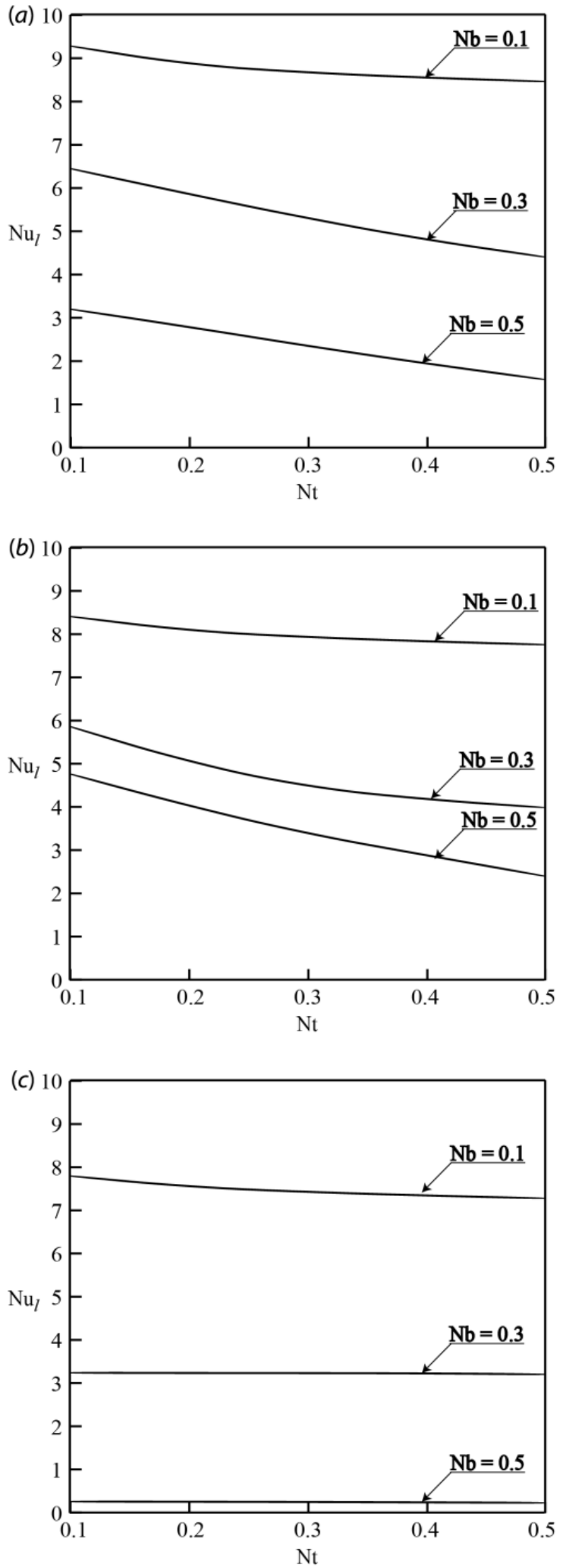

Figure 6. Variation of local Nusselt number as a function of $N t$ for $\operatorname{Pr}=6.2, L e=10, n=\xi=1, \varepsilon=0.5$ and $N b=0.1,0.3,0.5$ when (a) $H=-0.5$ (suction), (b) $H=0.0$ and (c) $H=0.5$

(injection)

Figure 6 illustrates the behavior of heat transfer rates for various values of $\mathrm{Nb}, \mathrm{Nt}, \mathrm{H}$ when $\operatorname{Pr}=6.2, \mathrm{Le}=10, n=\xi=1$ and $\varepsilon=0.5$. It is found that Nusselt number decreases when $N b$ and $N t$ increases. This observation is important because these parameters are directly influenced by the thermal enhancing factors. The influence of suction/injection 
parameter $H$ on local Nusselt number depicts that suction increases the heat transfer while injection decreases it when compared to the impermeable sheet. Further, it is worth mentioning to note that the trend is reversed in the case of suction for higher values of Brownian motion parameter $\mathrm{Nb}$. This circumstance happens due to the dominant effect of $\mathrm{Nb}$.
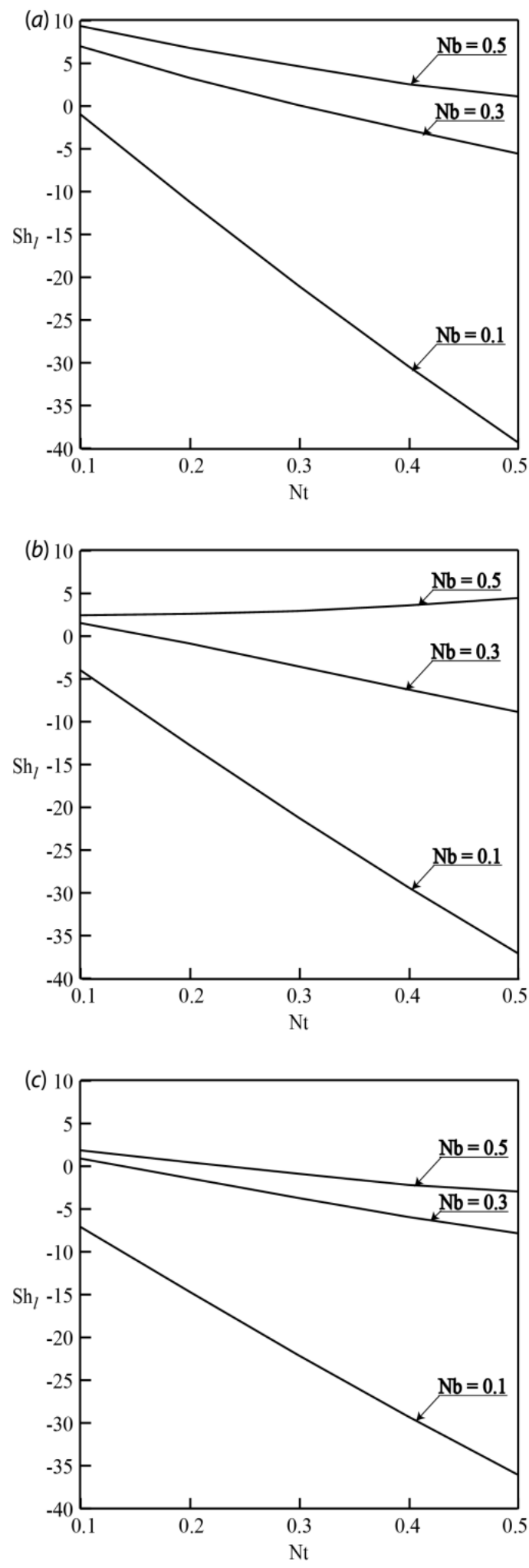

Figure 7. Variation of local Sherwood number as a function of $N t$ for $P r=6.2, L e=10, n=\xi=1, \varepsilon=0.5$ and $N b=0.1,0.3,0.5$ when (a) $H=-0.5$ (suction), (b) $H=0.0$ and (c) $H=0.5$

(injection)
The variation of mass transfer rates on diffusion parameters $(N b, N t)$ and suction/injection parameter $H$ when $P r=6.2$, $L e=10, n=\xi=1$ and $\varepsilon=0.5$ are shown in Figure 7. From these figures, it is clear that local Sherwood number increases with increasing Brownian motion parameter $\mathrm{Nb}$ but decreases as the thermophoresis parameter $N t$ increases. Consequently, suction increases and injection decreases the Sherwood number when compared to the case of impermeable sheet.

\section{CONCLUSIONS}

This paper presents the effects of velocity ratio, transverse magnetic field, suction/ injection, Brownian motion and thermophoresis on skin friction, temperature and concentration distributions, local Nusselt number and local Sherwood number in stagnation point flow towards a stretching sheet. The governing boundary layer equations of the problem are transformed into simple polynomial equations with the coefficients of independent parameters $\varepsilon, \xi, H, P r, n$, $L e, N b$, and $N t$. The results of the present study using GPDP is quite close to known numerical results. The advantage involved in the present variational technique is that the solution to the current problem is obtained with remarkable ease when compared to the tedious task of solving the governing boundary layer equations by other conventional methods. Also, one can note that the amount of calculation involved in the contemporary approach certainly less than that of other numerical procedures. Hence, the Governing Principle of Dissipative Processes, based on the sound physical reasoning is a powerful tool for obtaining a rapid solution to boundary layer flow, heat and mass transfer problems.

\section{REFERENCES}

[1] Hiemenz K. (1911). Die Grenzschicht an einem in den gleichförmigen Flüssigkeitsstrom eingetauchten geraden Kreiszylinder. Dingl. Polytech. J. 326: 321-410.

[2] Sakiadis BC. (1961). Boundary-layer behavior on continuous solid surfaces-II. The boundary layer on a continuous flat surface. American Inst. Chemical Eng. J. 7: 221-225. https://doi.org/10.1002/aic.690070211

[3] Crane LJ. (1970). Flow Past a Stretching Plate, Z. Angew. Math. Phys. 21: 645-647. https://doi.org/10.1007/BF01587695

[4] Bhattacharya DK. (1982). Application of Gyarmati's variational principle to laminar stagnation flow problem. Wärme-und Stoffübertragung 17: 27-30. https://doi.org/10.1007/BF01686962

[5] Ariel PD. (1994). Hiemenz flow in hydrodynamics, Acta Mechanica 103: 31-43. https://doi.org/10.1007/BF01180216

[6] Antony Raj S. (1987). A complete analytical solution to laminar heat transfer in axisymmetric stagnation point flow. Int. J. Heat and Mass Transfer 30: 2441-2444. https://doi.org/10.1016/0017-9310(87)90234-1

[7] Mahapatra TR, Gupta AS. (2002). Heat transfer in stagnation-point flow towards a stretching sheet. Heat and Mass Transfer 38: 517-521. https://doi.org/10.1007/s002310100215

[8] Ishak A, Jafar K, Nazar R, Pop I. (2009). MHD stagnation point flow towards a stretching sheet. Physica A 388: $3377-3383$. 
https://doi.org/10.1016/j.physa.2009.05.026

[9] Choi SUS. (1995). Enhancing thermal conductivity of fluids with nanoparticles in developments and applications of non-Newtonian flows, edited by D.A. Siginer, H.P. Wang. ASME FED 231/ MD 66: 99-105.

[10] Buongiorno J. (2006). Convective transport in Nanofluids. ASME J. Heat Transfer 128: 240-250. https://doi.org/10.1115/1.2150834

[11] Sharma PR, Singh G, Chamkha AJ. (2011). Unsteady heat transfer in steady stagnation point flow along stretching sheet in the presence of free stream. Int. J. Heat and Tech 29: 39-44.

[12] Bachok N, Ishak A, Pop I. (2013). Boundary layer stagnation-point flow toward a stretching/shrinking sheet in a nanofluid. ASME J. Heat Transfer 135: 1-5. https://doi.org/10.1115/1.4023303

[13] Ibrahim W, Shankar B, Nandeppanavar MM. (2013). MHD stagnation point flow and heat transfer due to nanofluid towards a stretching sheet. Int. J. Heat and Mass Transfer 56: 1-9. https://doi.org/10.1016/j.ijheatmasstransfer.2012.08.034

[14] Ibrahim W, Rizwan UH. (2015). Magnetohydrodynamic (MHD) stagnation point flow of nanofluid past a stretching sheet with convective boundary condition. J. Braz. Soc. Mech. Sci. Eng. 338: 1155-1164. https://doi.org/ 10.1007/s40430-015-0347-z

[15] Hamad MAA, Ferdows M. (2012). Similarity solution of boundary layer stagnation-point flow towards a heated porous stretching sheet saturated with a nanofluid with heat absorption/generation and suction/blowing: A Lie group analysis. Commun. Nonlinear Sci. Numer. Simulat. 17: $132-140$. https://doi.org/10.1016/j.cnsns.2011.02.024

[16] Reddy MG. (2014). Influence of thermal radiation on natural convection boundary layer flow of a nanofluid past a vertical plate with uniform heat flux. Int. J. Heat and Tech. 32: 1-7.

[17] Gyarmati I. (1969). On the governing principle of dissipative processes and its extension to non-linear problems. Ann. Phy. 23: 353-378. https://doi.org/10.1002/andp.19694780707

[18] Gyarmati I. (1970). Non Equilibrium Thermodynamics: Field Theory and Variational Principles. Springer-Verlag, Berlin, Germany.

[19] Onsager L. (1931). Reciprocal relations in irreversible processes-I. Phys. Rev. 37: 405-406. http://dx.doi.org/10.1103/PhysRev.37.405

[20] Onsager 1. (1931). Reciprocal relations in irreversible processes-II. Phys. Rev. 38: 2265-2266. http://dx.doi.org/10.1103/PhysRev.38.2265

[21] Prasad KV, Vajravelu K. (2009). Heat transfer in the MHD flow of a power law fluid over a non-isothermal stretching sheet. Int. J. Heat Mass Transfer 52: 49564965.

http://dx.doi.org/10.1016/j.ijheatmasstransfer.2009.05.0 22

\section{NOMENCLATURE}

$$
\begin{aligned}
& a, b, A \\
& \dot{a}_{i}
\end{aligned}
$$

proportionality constants

substantial variation of $i^{\text {th }}$ extensive transport quantity $a_{i}$

\begin{tabular}{|c|c|}
\hline$c_{f}$ & heat capacity of the base fluid \\
\hline$c_{p}$ & heat capacity of the nano particle \\
\hline$C$ & concentration of the nanofluid \\
\hline$C_{0}$ & $\begin{array}{l}\text { concentration of the nanofluid near the } \\
\text { stretching sheet }\end{array}$ \\
\hline$C_{\infty}$ & ambient concentration \\
\hline$d_{1}, d_{2}, d_{3}$ & $\begin{array}{l}\text { momentum, thermal and concentration } \\
\text { boundary layer thicknesses respectively }\end{array}$ \\
\hline$d_{1}{ }^{*}, d_{2}{ }^{*}, d_{3}{ }^{*}$ & $\begin{array}{l}\text { non dimensional momentum, thermal and } \\
\text { concentration boundary layer thicknesses } \\
\text { respectively }\end{array}$ \\
\hline$D_{B}$ & Brownian diffusion coefficient \\
\hline$D_{T}$ & thermal diffusion coefficient \\
\hline$H$ & suction/injection parameter \\
\hline$i, j$ & unit vector along $x$ and $y$ axes \\
\hline$J_{c}$ & mass flux \\
\hline$J_{q}$ & heat flux \\
\hline$J_{c}$ & mass current density \\
\hline $\boldsymbol{J}_{i}, \boldsymbol{J}_{\boldsymbol{k}}$ & fluxes in general \\
\hline$J_{q}$ & heat current density \\
\hline$l$ & representative length of the surface \\
\hline$L$ & Lagrangian \\
\hline Le & Lewis number \\
\hline$L_{c}, L_{s}, L_{\lambda}, L_{i k}$ & Conductivities \\
\hline$n$ & wall temperature parameter \\
\hline $\mathrm{Nb}$ & Brownian motion parameter \\
\hline$N t$ & thermophoresis parameter \\
\hline$N u_{l}$ & Nusselt number \\
\hline$P$ & hydro static pressure \\
\hline$P_{12}$ & momentum flux \\
\hline$\overline{\bar{P}}$ & pressure tensor \\
\hline $\begin{array}{l}P \\
o\end{array}$ & symmetrical part of second order viscous \\
\hline$\overline{\bar{P}} v s$ & pressure tensor of trace zero \\
\hline $\operatorname{Pr}$ & Prandtl number \\
\hline$R_{c}, R_{s}, R_{\lambda}, R_{i k}$ & Resistances \\
\hline$S h_{l}$ & Sherwood number \\
\hline$T$ & temperature of the nanofluid \\
\hline$T_{0}$ & non isothermal sheet temperature \\
\hline$T_{\infty}$ & ambient temperature \\
\hline$u$ & velocity of the fluid in $x$ direction \\
\hline$U_{0}$ & stretching sheet velocity \\
\hline$U_{\infty}$ & free stream velocity \\
\hline$v$ & velocity of the fluid in $y$ direction \\
\hline$v_{0}$ & suction/injection velocity \\
\hline$V$ & total volume \\
\hline $\boldsymbol{V}$ & velocity vector \\
\hline$X$ & coordinate along the plate \\
\hline $\boldsymbol{X}_{i}$ & forces in general \\
\hline & coordinate normal to the plate \\
\hline
\end{tabular}
externally imposed a magnetic field

\section{Greek symbols}

$\begin{array}{ll}\alpha & \text { thermal diffusivity of the fluid } \\ \alpha_{f} & \text { thermal diffusivity of the base fluid } \\ \delta & \text { variational symbol } \\ \delta_{i k} & \text { Kronecker delta } \\ \overline{\bar{\delta}} & \text { unit tensor } \\ \varepsilon & \text { velocity ratio parameter } \\ \phi & \text { non dimensional concentration } \\ \eta & \text { non dimensional coordinate normal to the } \\ \kappa & \text { plate } \\ \lambda & \text { electric conductivity } \\ & \text { thermal conductivity }\end{array}$


dynamic viscosity

kinematic viscosity

non dimensional temperature

mass density

entropy production

source density of the $i^{\text {th }}$ extensive transport quantity $a_{i}$

ratio of nano particle heat capacity and base fluid heat capacity

skin friction $\xi$

$\Psi, \Phi$

$\Gamma$

\section{Subscripts}

$\mathrm{p} \quad$ Nanoparticle

$\mathrm{f} \quad$ fluid (pure water) magnetic parameter

local dissipation potentials

state variable 